\title{
Transnational Engagements: From Debasement, Disability, and Disaster to Dignity-Stories of Menstruation Under Challenging Conditions
}

\author{
Edited by Milena Bacalja Perianes and Tomi-Ann Roberts
}

\section{INTRODUCTION}

While there has been a significant rise in attention paid to the menstrual experience, there are a variety of voices and bodies that remain inaudible and invisible. Those neglected are typically the most vulnerable and therefore less able to comfortably and confidently manage their menstrual health. The double stigma they face-as both menstruator and marginal-exacerbates their precarity and creates further boundaries to their health and wellbeing.

This chapter sheds light on the experiences of menstruators under a few such challenging conditions. Through the lens of three women, one living with disabilities, a second incarcerated and a third living in a disaster zone, we can begin to understand how they manage their menstrual needs. Their stories are a powerful (and at times disquieting) reminder of the need for equitable menstrual policies, diverse product design and more thoughtful and inclusive responses to women and girls at all stages, and in all situations.. Because situations constantly shift and evolve, innovative, inclusive and iterative responses must also be dynamic.

Ultimately, these first-person narratives challenge us to think about others muted in our current menstrual discourse and how we might bring them from margin to center. While we should be proud of the increased visibility of menstruation as an issue worthy of research and investment, we must redouble our efforts to be truly and profoundly inclusive. 


\section{From Behind the Wall: Menstruating While Incarcerated}

\section{Janelle Chambers}

Let me tell you a bit about myself. I am a 33-year-old mother of three children aged 2-13 and am happily married to a very loving wife. I had a very strong Christian upbringing and I love the Lord. I was raised in the church and had to deal with a lot in life including being constantly judged for my lifestyle. There were a lot of things that were not spoken about when I was young because it was not considered the "right speech." This has had a large impact on growing up, and I think, my experience of menstruating while being incarcerated.

No matter what a woman's upbringing, religious beliefs, sexual orientation, values or morals are, in life the one thing we have in common is menstruating every month regardless if we like it or not. So what is it like to have your period while incarcerated? The one thing we as women all have in common is that periods suck! It sucks even more when you are in a place you never dreamed of being. So not only do you have a million and one thoughts racing through your head, being fearful of the unknown and unexpected, but then BAM here comes Aunt Flo to visit.

When your period arrives you suddenly find yourself in a room with a toilet that is shared with another woman watching you. Are there tampons or pads under the bathroom sink ... umm I think not, this is jail. The door is locked so what do you do? Do you bleed everywhere or wrap toilet paper up and lay it on your panties? Sometimes incarcerated women choose to lay toilet paper in their underwear until the dayroom opens. That's if it does.

Managing your menstruation is a very public experience when incarcerated. In order to get some no-name brand sanitary napkins women have to be good. We can only access products in the dayroom, which is a shared space were inmates congregate and where necessary or luxury items are purchased or distributed. Everyone knows when you are on your period because your only option to get a product is to go to the dayroom to request them, then take them back to your cell. How embarrassing! Imagine, being with hundreds of women you don't know. You don't know how many of them will have an off day that may lead to a fight. You don't know who will be making comments among themselves about the "bleeding inmate." There are deputies that like to belittle you and treat you like a caged animal. We have to learn to be okay with it, to be desensitized to this kind of treatment-this is the price we pay for the crimes we committed. There is a lot of shame here. "Oh the convict needs a pad for her bloody period," the guards broadcast to the whole dayroom. The way we are treated makes us feel more shame than for what we have already done.

To make matters worse the dayroom visit usually takes up your time so you can't shower, which means everyone remembers you as the stinky bloody chick that didn't get to take her ( 5 minute) shower when the dayroom was 
open. When you are back in your cell you have to hope your roommate is cool and gives you space to deal with it.

I was fortunate when I was first incarcerated to have good roommates. They each taught me valuable things that helped me on my journey while in jail. Learning how to manage your period was a part of that. I learned how to wash up in the small sink in our room that was connected to the toilet. This same sink was used to brush our teeth, wash our face and hands. We had a routine when one of us got our period which helped. We were lucky to have a good rapport with each other. An inmate was even luckier when they developed a good relationship with one or more deputies to be able to shower when other inmates were sleeping.

Hygiene was a big concern for me, for us all. Of course problems arise when we have low-quality products, and limited access to water to manage our menstruation. I believe my fibroids were caused by my incarceration and having to use things no woman should ever have to use. Who thinks of the health risks at a time and place like that? All I was concerned about was having my hygiene up to par and not having a bloody mess. As we only get to change out of our county blues once a week, it's important to keep them as clean as possible. Can you imagine if you had an accident from Aunt Flo and you now have to wait a week for a clean pair of clothes? With only one pair of panties to hold your napkin in place, you have to be careful.

Menstruation is an ongoing issue you regularly have to handle. Think about it. It's your first court hearing, now you're thinking, “yes! I finally get a break. I can breathe some fresh air and hopefully see my family members, or use the phone in the holding tank." Of course that sounds fantastic until reality hits you when you get off the bus from court back at the jailhouse. You are going to be strip-searched, but you have your period.

The strip search process when you reenter jail after a court hearing is particularly humiliating. They always want to make sure you are not bringing anything back into the facility after having contact with the outside world. Sometimes they line up 100 women and make all of us remove our clothes and stand next to each other, in the nude, being degraded and humiliated. Now you are told to run your dirty hands through your hair. Lift up your breasts while the deputy runs their hands underneath. They tell you to run in place to see if anything will fall from anywhere. They make you bend over, squat and cough. They tell you to take out anything in your Va-jay-jay, like pads or tampons, and throw it in front of you in the center of the room where all can see it.

So the center of the room becomes one big open trash bin of bloody products that some of the inmates have to later clean up. "Nasty, dirty, filthy pigs" was a famous line the deputies would say to us. "You $\mathrm{B}^{* * * * *}$ stink, no wonder you're here because nobody out there wanted you." It never matters who you were, us women inmates were treated the same. Not only was our freedom taken from us, but now our dignity ripped right from underneath us. Some women there had never even been intimate with another person and 
then were forced to expose their most sacred place. We didn't mean anything so why should anyone care. I stood next to a nun once and all she could do was cry and repeat The Lord's Prayer. If you were menstruating it didn't matter if blood just ran down your legs while you stood there. You just wanted the humiliation and nightmare to end and hope nobody remembered what you looked like.

I can probably sit here and tell you of the 1546 days of torment and torture I endured, but this is a very hard state of mind to go back to that I have spent many years dealing with, on top of my many other medical issues. I really hope and pray that I have shed some light on the many different emotional effects of dealing with menstruating while being incarcerated. I am grateful to be able to be the voice of so many of my sisters who have, and who are still, dealing with these challenges. We now have a voice for us that may be heard a little better with the help of this book.

\section{Designing Menstrual Products for Disabled Bodies}

\section{Jane Hartman Adamé}

It somehow slips society's mind that disabled people can menstruate, be considered sexually attractive, have sex, and even reproduce. Of course, some of these are not true of all disabled people, but neither are they true of all able-bodied individuals. All of these same complexities that an able-bodied person can experience are also possible for disabled individuals like myself.

Adding to the complexity of menstruating with a disability is that products that are made to manage and support our menstrual health and wellness are often made without any consideration of our specific experiences. As a result, it can be a struggle to find products to meet your individual needs. Luckily, changes are happening in this arena, with more innovative, unique, problem-solving products arriving to market at an increasing pace. However, we have a lot of ground to make up for.

When we consider the concept of disability and menstruation, one often-neglected concept is that menstruation itself looks a lot like disability. I'm not speaking just about dysmenorrhea, or endometriosis, although those certainly can qualify as well-but even the "simple, normal" menstrual cycle. To wrap our heads around this, let's look at two common definitions of disability, the medical model and the social model.

As a medical condition, menstruation requires intervention with (usually) approved medical devices to manage the collection of the menses itself. It can also require care and treatment for the symptoms that come along, such as pain and inflammation, by over-the-counter pain meds, warm compresses or packs, or pain creams applied to the lower abdomen or even the low back.

In a more social context, a menstruating person may need to alter the clothes they wear for the day to accommodate bloating and provide comfort, which could alter how they are perceived (or feel they are perceived) 
outside of the home. They may need to miss a day or two of work with severe cramps, or heavy bleeding that requires frequent product changes. These frequent bathroom trips or distracting bouts of pain can inhibit job performance, eat up sick days, and have a myriad of other impacts on a person's life. Due to the cultural shame and taboo surrounding the period, attempts are made to hide these changes, such as tucking one's tampon into a sleeve to slip unnoticed into the bathroom. Additionally, there is social pressure from advertisements that a person should be able to go forth and be productiveperiod or not. There is also pressure, due to the normalization of this process, to not seek medical care for menstrual issues. It's seen as something common, but even common conditions can have significant impacts on us.

The menstrual cycle, when viewed through the lens of disability, is a relapsing, recurring condition (in that menstruation itself and its uncomfortable symptoms occur approximately monthly for a number of days, and then recede for the remainder of the month) that just so happens to occur with such prevalence and regularity that its very essence is dismissed, and complaints about changing symptoms can be overlooked or ignored. Even just the fear of the possibility of being dismissed (in addition to the shame and taboo aspects of this area of our health) keeps many of our menstruating community from setting foot in a doctor's office to talk about these issues.

Evidence of delayed diagnoses of endometriosis and other serious life-affecting menstrual conditions shows us that menstruation is not taken seriously. When you add menstruation to disability, then you have a double whammy. Women's pain is expected, from all processes from typical menstruation, to atypical menstruation, to childbirth and beyond. If we allowed ourselves to look at the taboo concept of menstruation with the lens of the even more taboo concept of disability, we could start to treat both of these states of being with more care, both medically and socially.

Although I can't speak for all disabilities, living with hypermobile Ehlers-Danlos Syndrome certainly poses some challenges when it comes to managing my menstrual cycle. Chronic pain, alone, can be greatly exacerbated by menstrual pain. Add to that the risk factor of changing out menstrual products with dislocation risk, and it's clear that periods are extra challenging to manage. Another challenge that my disability poses, and which is certainly not unique to me, is pelvic floor dysfunction. Whether you have a weakened pelvic floor, prolapse, vaginismus, or other eccentric pelvic muscle activation, internally worn products can be a pain to use. Anything requiring bearing down (applying downward force to the pelvic floor by way of muscle activation) can be a risky thing to do if the pelvic floor is weakened or any organs are prolapsed. If muscles are overly tight, internally worn products can be uncomfortable to wear or pose a serious challenge when it comes to removal.

Outside of my personal experience, I've also come to learn of the challenges faced by folks who utilize carers to manage their menstrual hygiene. This can be a challenging and sensitive subject, as the carer has to 
directly interact with the user's menses. Of course, menstrual products are not made to be managed by anyone other than the user, which is where we find ourselves having to get creative.

Fortunately, many of us don't have to be relegated to pads-although there is nothing inherently bad about a pad. It's my belief that all menstrual care options are good options, and nobody other than you can decide what the right choice is for your body.

Personally, I do prefer internally worn products, especially those that require less frequent changes than tampons. Menstrual discs and cups can both be worn for up to 12 hours-even if you can wear your tampons for the maximum amount of time, which is 8 hours, these newer options cut down the number of product changes per day from 3 to 2 . This may seem like an insignificant difference, but if you have mobility challenges, fatigue, or chronic pain, one less change per day can mean the ability to use that energy for something else.

When I lost the ability to use my menstrual cup, I was devastated. For myself and many others, the complicated maneuver required to remove a cup makes it nearly impossible (or very risky) to use. Many times, I subluxed or dislocated joints and/or set muscles into spasm, all from trying to position my body and arm in such a way that I could grasp the cup to break the seal in order to safely remove it. Instead of admitting defeat, I decided to create a cup that I and others like me could use.

With my design, now known as the FLEX Cup, I added a ReleaseRing ${ }^{\mathrm{TM}}$ which is a piece that threads from the top of the cup through the middle, ending in a soft silicone ring that moves with your body when worn, so the cup can be worn low with the ring accessible in the labial area, or more internally. Either way, since this piece is attached to the top of the cup, it indents the side when pulled and makes cup removal more akin to removing a tampon.

When we launched the cup on Kickstarter, it made huge waves-many people never considered the challenges that those of us with disabilities faced during menstruation. Others who were nervous to try cups found ease in this design. With many people, both disabled and able-bodied, the risk of potentially ending in the ER to have the cup removed was a deal breaker. I knew that eliminating this risk would be of great benefit to so many people. During the Kickstarter, The Flex Company saw value in our design and eventually bought the patent. They also added my cofounder Andy Miller and myself to their team, and together we are making great strides to make menstrual products comfortable and accessible to all, having landed on retail shelves just about a year after inception.

The other product we make, the menstrual disc, can be a great option and is a top choice for those with Ehlers-Danlos Syndrome and other mobility-limiting conditions. Since insertion doesn't require any complex folding or grip strength, insertion of a disc can be easier than a cup for some. Since this product can also be worn for up to 12 hours but through its single use, it eliminates the cleaning process, which can be taxing for many. 
Additionally, the FLEX menstrual disc is the only disc available on a subscription cycle-we don't usually consider the process of going to the store to restock menstrual products as an access issue, but it is. Here, reusable options or subscription services can be great access solutions.

So, how do you determine what is the right solution for your body? I know many people flock to YouTube to watch reviews of menstrual products, but in truth, there is so much variance in our anatomies, regardless of additional conditions and factors, that there is only one true way to determine what will work best for you — and that is to give new things a try.

\section{Menstruation in Emergencies: Developing a Period-Friendly EMERGENCY RESPONSE}

\section{Mayuri Bhattacharjee}

I am Mayuri Bhattacharjee, a menstrual health educator with experience of teaching more than 8000 women and girls about menstrual health in India, especially in the flood-affected regions in Assam, a state in India's northeast. I run a nonprofit which works on the intersections of public health and climate action and I am a Climate Reality Leader at the Climate Reality Project.

I grew up in Assam, India. A state which faces floods every year and when I attained menarche, I, like many girls in India, was subjected to menstrual taboos which made me feel impure. So, now to change this narrative, I work at the crossroads of natural disasters and menstrual health because of my own background and lived experience in Assam.

Women and adolescent girls in disaster-prone and fragile contexts face many challenges and Assam's floods are no exception to this. As you might have seen in news covering disasters, toilets, and other sanitation and hygiene facilities are hard hit during floods across the world. Menstrual hygiene practices are often compromised in the tough conditions surrounding a natural disaster. This is in part due to a lack of proper shelter, water supply, disposal and washing facilities for women and girls to safely and securely manage their periods.

In Assam, a lack of knowledge around the biological process of menstruation has exacerbated negative experiences around menstruation. Many myths exist around the topic including the common notion that menstruation is the passing of bad blood from a woman's body. In some communities, the practice of segregation during menstruation is observed even in a flood shelter. This is a risky practice because being separated during floods can expose those menstruating to health and security risks. In a worstcase scenario, kidnapping or human trafficking can occur as floods are a fertile ground for traffickers who are on the lookout for easy prey. Apart from physical risks, the notion that menstrual blood is impure and so is a menstruating woman, puts unwanted mental stress on women who are already carrying a significant burden in emergency situations. 
Fundamentally, more attention needs to be paid to the intersection between climate change and women's changing health needs. While a wide variety of information and materials are being made available to women regarding menstruation, the experiences of women in emergencies remain an underserved topic.

With climate change increasingly impacting weather patterns, like many other places, annual flooding in Assam will continue to be a challenge. As researchers and practitioners, we need to think about how to address women's and girl's menstrual health needs in this context and prepare them to be able to manage their menstrual cycles during emergency situations. This is a public health issue often overlooked by local authorities. This should not be an afterthought during a crisis, rather governments and public bodies need to work on mitigation strategies which target women's specific health needs. In Assam, this can include pre-disaster planning and interventions in education and infrastructure including the construction of season embankments, and disaster specific plans for the disposal of menstrual waste. Menstrual waste disposal is a challenge even in normal situations and the problem is only exacerbated during floods.

As a result of this lack of planning or foresight from state governments, there is a tendency to apply a Band-Aid or a one size fits all approach to menstrual health gaps. Most often that solution is the blanket distribution of sanitary pads. This process often leads to a glamorization of pads and the demonization of local cloth alternatives. Rarely, are tampons or menstrual cups a part of hygiene kits because of the taboos around virginity and limited knowledge among those procuring and distributing relief materials. Instead of promoting only one product - usually non-compostable, disposable padsgovernment and humanitarian agencies should look at other eco-friendly options such as low-cost cloth pads and menstrual cups. Thoughtful and considered emergency responses remain a barrier to adequate menstrual health in emergency settings because menstrual health remains a women's issue, a minor issue, and not a public health concern.

I believe an important first step toward developing a period-friendly emergency response is to normalize conversations around menstruation especially among those who plan mitigation and emergency responses. We must move the conversation from just women and adolescent girls, but ensure men are also empowered to talk about menstruation without shame. The most effective step toward this is menstrual health education at a community-level and special orientation sessions for frontline humanitarian response workers.

Addressing menstrual health needs in contexts in which natural disasters occur regularly, requires a sensitive response to women's diverse experiences. At the Sikun Relief Foundation, we don't believe in promoting one kind of menstrual hygiene product, but we believe in informed choice. Women in emergency settings deserve the same choices as every other woman therefore our interventions focus on introducing a basket of menstrual hygiene products and giving women the knowledge they need to make informed decisions 
for the context they are in. Unlike traditional menstrual health education, our interventions start well before the flood season with a major focus on how women and girls can understand and manage their cycles before and during an emergency. Rather than dictating one solution, we believe in women and girls coming up with their own personal strategies to deal with their periods.

Co-creating WASH and MHM solutions and frameworks together with women and adolescent girls would be more effective in developing a gender-sensitive response mechanism during emergencies because women's experiences are central to the effectiveness of disaster management planning and response.

Open Access This chapter is licensed under the terms of the Creative Commons Attribution 4.0 International License (http://creativecommons.org/licenses/ by $/ 4.0 /$ ), which permits use, sharing, adaptation, distribution and reproduction in any medium or format, as long as you give appropriate credit to the original author(s) and the source, provide a link to the Creative Commons license and indicate if changes were made.

The images or other third party material in this chapter are included in the chapter's Creative Commons license, unless indicated otherwise in a credit line to the material. If material is not included in the chapter's Creative Commons license and your intended use is not permitted by statutory regulation or exceeds the permitted use, you will need to obtain permission directly from the copyright holder.

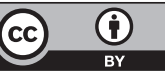

